\title{
The Crystal Structure of Rubidium Triselenocyanate Hemihydrate
}

\author{
S VER R H A U GE \\ Chemical Institute, University of Bergen, Bergen, Norway
}

\begin{abstract}
The crystal structure of rubidium triselenocyanate hemihydrate, $\mathrm{Rb}(\mathrm{SeCN})_{3} \cdot \frac{1}{2} \mathrm{H}_{2} \mathrm{O}$, has been determined by $\mathrm{X}$-ray methods, and refined by full-matrix least squares procedures. The crystals are orthorhombic, space group $F m 2 m$ (No. 42), with $a=16.710(8) \AA$, $b=4.833(3) \AA, c=22.808(10) \AA$, and eight formula units per unit cell.

In the crystals, the triselenocyanate ions lie across crystallographic mirror planes. The three-selenium sequence of the triselenocyanate ion is nearly linear, with $\mathrm{Se}-\mathrm{Se}-\mathrm{Se}$ bond angle $176.9(3)^{\circ}$, and $\mathrm{Se}-\mathrm{Se}$ bond lengths of $2.656(3) \AA$, which is $0.32 \AA$ longer than single covalent selenium-selenium bonds.

Each of the selenocyanate groups is linear within the error. The terminal selenocyanate groups and the middle selenium atom, $\mathrm{Se}_{2}$, lie nearly in the same plane. This plane makes an angle of $65.0^{\circ}$ with a plane through the middle selenocyanate group and the terminal selenium atoms.
\end{abstract}

$T^{\mathrm{T}}$ The triselenocyanate ion was first isolated by Verneuil ${ }^{\mathbf{1}}$ in the form of the potassium salt. The crystal structure of the potassium salt, a hemihydrate, has been determined. ${ }^{2}$ Birckenback and Kellermann ${ }^{3}$ prepared the cesium salt. Later, rubidium triselenocyanate hemihydrate, $\mathrm{Rb}(\mathrm{SeCN})_{3} \cdot \frac{1}{2} \mathrm{H}_{2} \mathrm{O}$, was isolated. ${ }^{4}$ Its crystal structure analysis, reported here, has been carried out as part of a study of linear three-selenium systems.

\section{CRYSTAL DATA}

Preparative and crystallographic data on rubidium triselenocyanate hemihydrate have been reported earlier. ${ }^{4}$

The salt, $\mathrm{Rb}(\mathrm{SeCN})_{3} \cdot \frac{1}{2} \mathrm{H}_{2} \mathrm{O}$, forms long, brown, orthorhombic prisms elongated along the $b$ axis, with $a=16.710(8) \AA, b=4.883(3) \AA$, and $c=22.808(10) \AA$. The unit cell dimensions were determined from zero-layer Weissenberg photographs around the $b$ and $c$ axes, and evaluated by means of a least squares program. 
There are eight formula units per unit cell; density, calc. 2.95, found $2.96 \mathrm{~g} / \mathrm{cm}^{3}$. The space group, from systematic absences and subsequent structure analysis, is $F m 2 m$ (No. 42 ).

Intensities were estimated visually from integrated Weissenberg photographs around the $b$ and $c$ axes, taken with $\mathrm{Cu} K \alpha$ radiation using the multifilm technique. The three-dimensional refinement was based on the $h 0 l-h 3 l$ and $h k 0-h k 1$ data. 119 out of $132 h 0 l, 115$ out of $117 h 1 l, 113$ out of $120 h 2 l$, 88 out of $93 h 3 l, 31$ out of $32 h k 0$, and 23 out of $26 h k l$ reflections, in all 429 out of 455 independent $h 0 l-h 3 l$ and $h k 0-h k 1$ reflections, accessible with $\mathrm{Cu} K \alpha$ radiation, were observed with measurable intensities. Three different crystals with approximately prism shape were used; the crystal used for the collection of the $h 0 l$ data had cross-section $0.05 \times 0.11 \mathrm{~mm}^{2}$ and length $0.11 \mathrm{~mm}$. The corresponding dimensions of the crystals used for the $h 1 l-h 3 l$ and for the $h k 0-h k l$ data were $0.09 \times 0.08 \mathrm{~mm}^{2}$ and $0.125 \mathrm{~mm}$, and $0.08 \times 0.13 \mathrm{~mm}^{2}$ and $0.08 \mathrm{~mm}$, respectively. The linear absorption coefficient, $\mu=232 \mathrm{~cm}^{-1}$.

The intensities were corrected for absorption by the method of Coppens et al. ${ }^{5} \mathrm{~A}$ sub-division of 10,4 , and 14 Gaussian points along the $a, b$, and $c$ axes, respectively, were used for all three crystals.

The computer programs used for data reduction and absorption correction were made available by the Chemical Department of X-Ray Crystallography, Weizmann Institute of Science, Rehovoth, Israel, and modified for use on the IBM 360/50H computer by Dr. D. Rabinovich.

\section{THE STRUCTURE ANALYSIS}

With eight rubidium ions and eight triselenocyanate ions in the unit cell, and four water molecules, the space group $F m 2 m$, if correct, would demand that the ions, and the water molecule, lie in special positions. The structure of the potassium salt, which has space group $F 2$ and similar although monoclinic unit cell, indicated that in the rubidium salt, the triselenocyanate ion may lie across a mirror plane, with the middle selenocyanate group in the plane. This was confirmed through the structure analysis.

Approximate $x$ and $z$ coordinates of the rubidium ion and of the selenium atoms were found from the $h 0 l$ Patterson map, by reference to the structure of $\mathrm{K}(\mathrm{SeCN})_{3} \cdot \frac{1}{2} \mathrm{H}_{2} \mathrm{O}^{2}$ The carbon and nitrogen atoms were placed partly on the basis of subsequent $h 0 l$ Fourier maps, and partly from the analogy with the potassium salt.

The three-dimensional refinement was carried out on the IBM $360 / 50 \mathrm{H}$ computer, using a full-matrix least squares program with weighting scheme $1 /\left(1+A F_{o}+B F_{0}{ }^{2}\right)$. The values used for $A$ and $B$ were $-1.2 \times 10^{-2}$ and $6 \times 10^{-5}$, respectively. Refinement with isotropic temperature factors brought the reliability index, $R$, to 0.111 . The observed structure factors were then corrected for secondary extinction by the method of Zachariasen, ${ }^{6}$ neglecting the absorption term, since this correction had been carried out earlier: $F_{\text {corr }}=K F_{\mathrm{o}}\left(1+\beta C I_{\mathrm{o}}\right)$, where $\beta=2\left(1+\cos ^{4} 2 \theta\right) /\left(1+\cos ^{2} 2 \theta\right)^{2}$, and $C$ was found to be $1.1 \times 10^{-7}$. The intensities of reflections that occurred more than once in the data set, were averaged, and such reflections thereafter included only once. 
The final refinement, based on the corrected observed structure factors, and with anisotropic temperature factors for rubidium and selenium, brought the reliability index, $R$, down to 0.083 , with unobserved reflections included if $\left|F_{\mathrm{c}}\right|$ exceeds the observable limit.

The programs used for the least squares refinement, extinction correction, calculation of distances and angles, and least squares planes, were written by K. Maartmann-Moe of this Institute.

The calculated structure factors were based on atomic scattering factors, given in the International Tables (Ref. 7, Table 3.3.1A). The scattering factors for selenium and rubidium were corrected for anomalous dispersion, real and imaginary parts (Ref. 7, Table 3.3.2A), by taking the amplitude of $f$ as the corrected value.

The final atomic coordinates and temperature parameters are listed in Tables 1 and 2, and the structure factors in Table 3.

Table 1. Atomic coordinates for rubidium triselenocyanate hemihydrate, in fractions of orthorhombic cell edges, with origin on $m 2 m$. Isotropic temperature parameters $\left(\AA^{2}\right)$ in the form $\exp -\left[B\left(\sin ^{2} \theta / \lambda^{2}\right)\right]$. Standard deviations from least squares are given in parentheses.

$\begin{array}{lcccc} & x & y & z & B \\ \mathrm{Rb} & 0.14340(20) & 0.2384(17) & 0 & \\ \mathrm{Se}_{1} & 0.15892(12) & 0.0128(14) & 0.19247(10) & \\ \mathrm{Se}_{2} & 0 & 0 & 0.19401(14) & \\ \mathrm{C}_{1} & 0.1688(11) & -0.165(5) & 0.1230(9) & 2.5(4) \\ \mathrm{C}_{2} & 0 & 0.232(10) & 0.1310(14) & 3.3(6) \\ \mathrm{N}_{1} & 0.1768(11) & -0.276(7) & 0.0778(9) & 4.1(4) \\ \mathrm{N}_{2} & 0 & 0.378(6) & 0.0905(12) & 3.2(5) \\ \mathrm{O} & 0 & -0.147(9) & 0 & 4.7(9)\end{array}$

Table 2. Anisotropic temperature parameters $\left(\AA^{2}\right)$ in the form $\exp -\left[B_{11}\left(h^{2} / 4 a^{2}\right)+\cdots+B_{23}(k l / 4 b c)+\cdots\right]$. Standard deviations are given in parentheses.

\begin{tabular}{llllccc} 
& \multicolumn{1}{c}{$B_{11}$} & $B_{22}$ & $B_{33}$ & $B_{23}$ & $B_{13}$ & $B_{12}$ \\
$\mathrm{Rb}$ & $3.88(11)$ & $2.89(18)$ & $3.95(12)$ & 0 & 0 & $-0.38(17)$ \\
$\mathrm{Se}_{1}$ & $2.73(7)$ & $4.86(18)$ & $3.08(8)$ & $-0.48(14)$ & $-0.26(8)$ & $-0.01(13)$ \\
$\mathrm{Se}_{2}$ & $3.44(11)$ & $2.94(19)$ & $2.66(10)$ & $-0.07(18)$ & 0 & 0
\end{tabular}

\section{THE TRISELENOCYANATE ION}

Bond lengths and angles in the triselenocyanate ion, based on the atomic coordinates in Table 1, are listed in Table 4. The uncertainties in cell dimensions are taken into account in the given standard deviations. In Table 4, a prime denotes an atom located at $(\bar{x}, y, z)$ relative to the unprimed one; the primed atom is related to the unprimed one through the operation of the mirror plane parallel to (100) passing through the middle selenium atom, $\mathrm{Se}_{2}$.

In the triselenocyanate ion, the $\mathrm{Se}-\mathrm{Se}-\mathrm{Se}$ angle is $176.9(3)^{\circ}$, and the three-selenium sequence is thus very nearly linear. The Se-Se bond length is $2.656(3) \AA$. In contrast to the present salt, where mirror plane symmetry makes the two $\mathrm{Se}-\mathrm{Se}$ bonds equal, the three-selenium sequence in the corre-

Acta Chem. Scand. 25 (1971) No. 8 
Table 3. Observed and calculated structure factors. Unobserved reflections are indicated by a minus sign on $F(O)$.

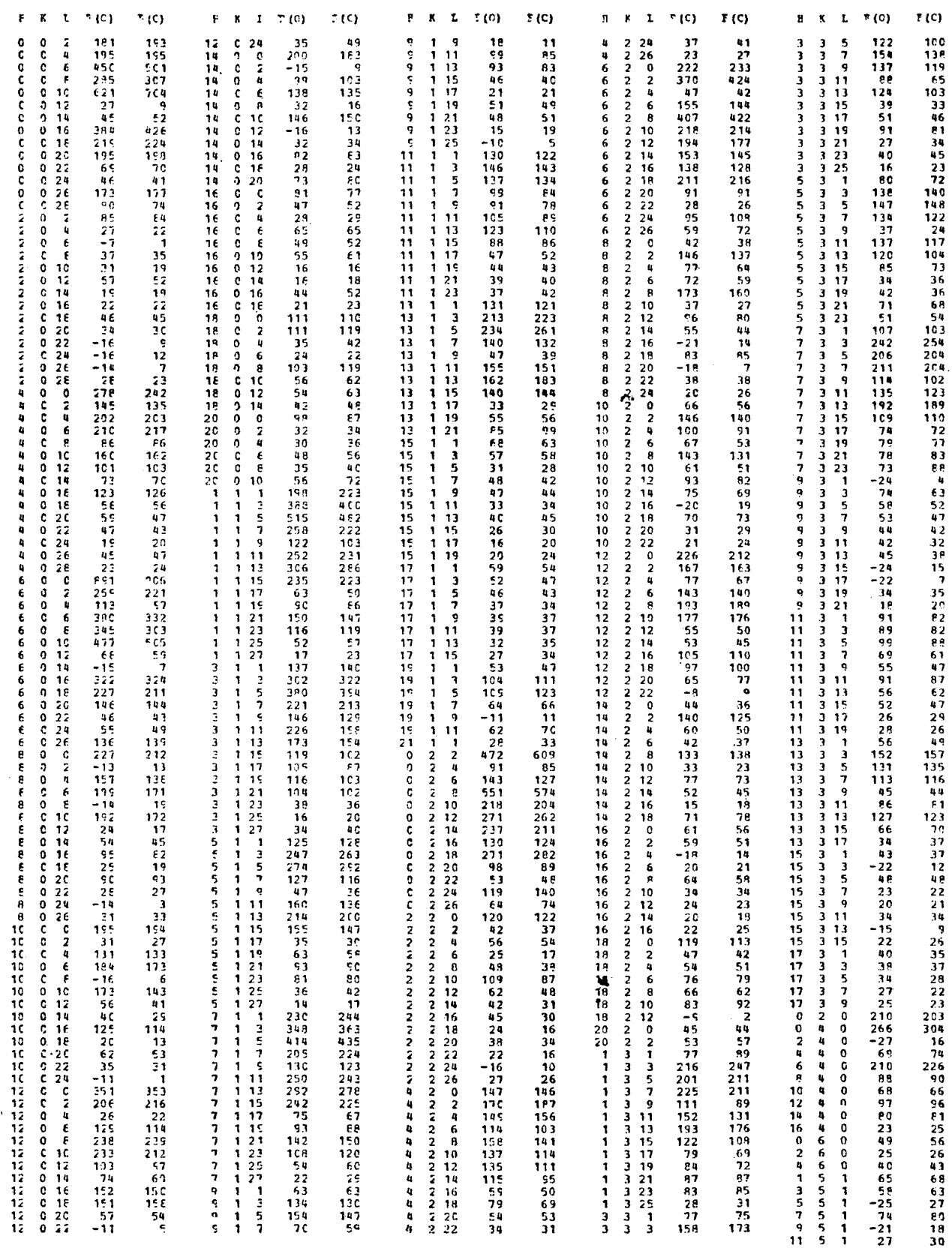

Acta Chem. Scand. 25 (1971) No. 8 
Table 4. Dimensions of the triselenocyanate ion. Bond lengths $(\AA)$ and angles $\left({ }^{\circ}\right)$. Standard deviations are given in parentheses.

$$
\begin{aligned}
& \mathrm{Se}_{1}-\mathrm{Se}_{2}=2.656(3) \\
& \mathrm{Se}_{1}-\mathrm{C}_{1}=1.81(2) \\
& \mathrm{Se}_{2}-\mathrm{C}_{2}=1.82(4) \\
& \mathrm{C}_{1}-\mathrm{N}_{1}=1.17(3) \\
& \mathrm{C}_{2}-\mathrm{N}_{2}=1.16(5)
\end{aligned}
$$

$$
\begin{aligned}
& \angle \mathrm{Se}_{1}-\mathrm{Se}_{2}-\mathrm{Se}_{1}{ }^{\prime}=176.9(3) \\
& \angle \mathrm{Se}_{1}-\mathrm{C}_{1}-\mathrm{N}_{1}=178.4(17) \\
& \angle \mathrm{Se}_{2}-\mathrm{C}_{2}-\mathrm{N}_{2}=179.3(30) \\
& \angle \mathrm{Se}_{1}-\mathrm{Se}_{2}-\mathrm{C}_{2}=88.58(15) \\
& \angle \mathrm{Se}_{2}-\mathrm{Se}_{1}-\mathrm{C}_{1}=95.3(8)
\end{aligned}
$$

sponding potassium salt ${ }^{2}$ is slightly unsymmetrical, with $\mathrm{Se}-\mathrm{Se}-\mathrm{Se}$ bond angle of $176.0^{\circ}$ and Se-Se bond lengths of 2.689 and $2.648 \AA$. The total length of the three-selenium sequence (the sum of the lengths of the two bonds) is 5.337 $\AA$ in the potassium salt and 5.312 $\AA$ in the rubidium salt, the mean

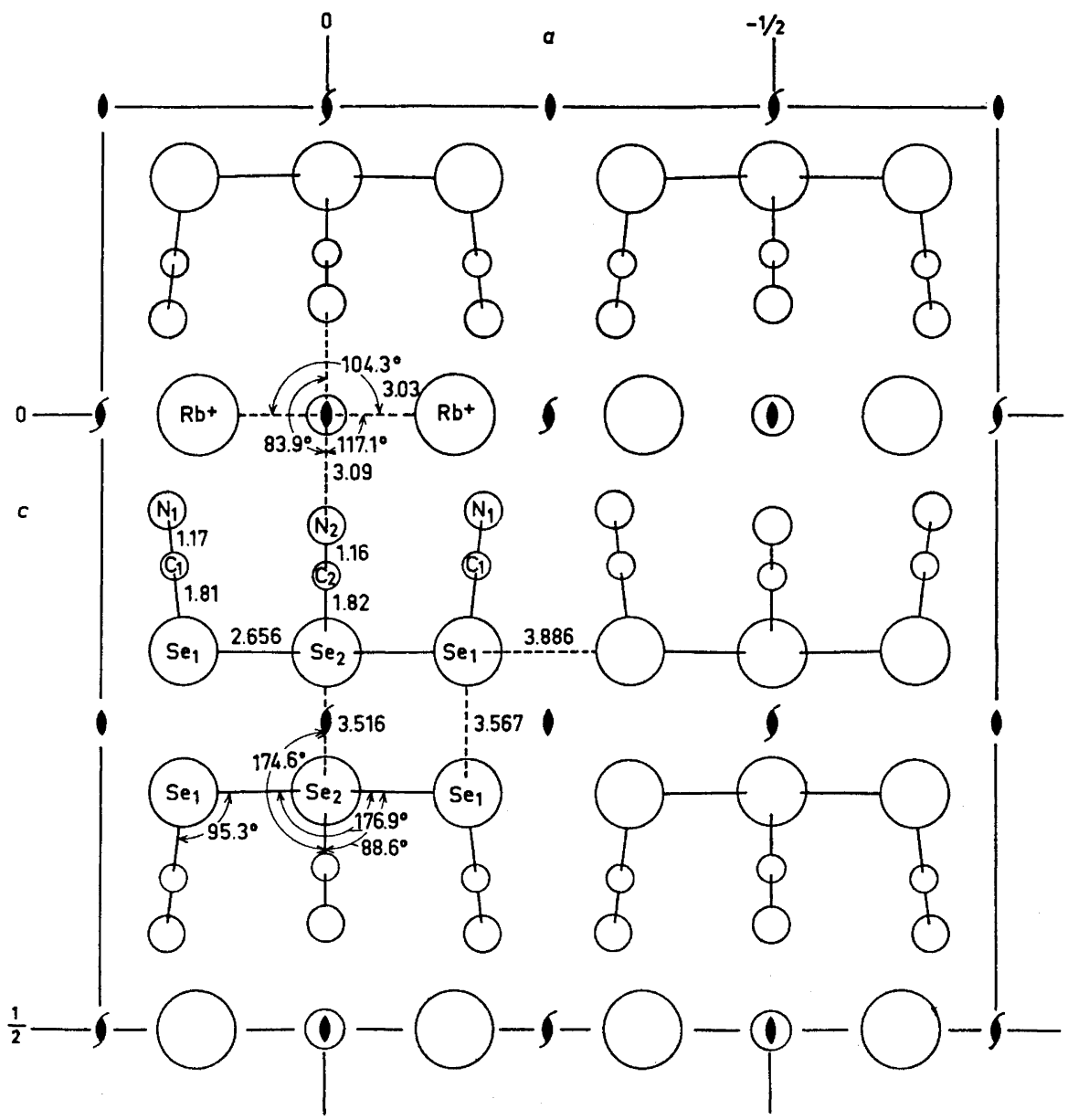

Fig. 1. Rubidium triselenocyanate hemihydrate as seen along the $b$ crystal axis. Acta Chem. Scand. 25 (1971) No. 8 
value being $5.325 \AA$. The same mean total length is found for the very nearly linear, unsymmetrical three-selenium sequence in two salts of the triselenourea ion, ${ }^{8}$ the dichloride and dibromide.

Thus, from four structure analyses of linear three-selenium systems, one symmetrical and three unsymmetrical, it is found that the mean value of the $\mathrm{Se}-\mathrm{Se}$ bond length is $2.66 \AA$. This is $0.32 \AA$ longer than a single covalent selenium-selenium bond. ${ }^{9}$

The $\mathrm{Se}-\mathrm{Se}-\mathrm{C}$ bond angle at the middle selenium atom, $\mathrm{Se}_{2}$, is $88.58(15)^{\circ}$, and the $\mathrm{Se}-\mathrm{Se}-\mathrm{C}$ bond angle at the terminal selenium atom, $\mathrm{Se}_{1}$, is $95.3(8)^{\circ}$.

Each of the selenocyanate groups of the ion is linear within the error; the found values for the $\mathrm{Se}-\mathrm{C}-\mathrm{N}$ angles are $178.4(17)^{\circ}$ and $179.3(30)^{\circ}$. The $\mathrm{Se}-\mathrm{C}$ bonds are 1.81(2) and 1.82(4) $\AA$, and the $\mathrm{C}-\mathrm{N}$ bonds $1.17(3)$ and 1.16(3) $\AA$. Within the accuracy of the analyses, these values are the same as found in the corresponding potassium salt, ${ }^{2}$ and also for the selenocyanate ion in the crystals of potassium selenocyanate. ${ }^{10}$

The atoms of the terminal selenocyanate groups and the middle selenium atom, $\mathrm{Se}_{2}$, are approximately co-planar, the largest deviation of an atom from a least squares plane being $0.05 \AA$ ( $c f$. Table 6). So are the atoms of the middle selenocyanate group and the terminal selenium atoms, $\mathrm{Se}_{1}$ and $\mathrm{Se}_{1}{ }^{\prime}$, the largest deviation of an atom from a least squares plane being $0.02 \AA$. These two planes make an angle of $65.0^{\circ}$ with each other.

Tables 5. Close interionic selenium-selenium contacts. Distances $(\AA)$ and angles $\left({ }^{\circ}\right)$. Standard deviations are given in parentheses.

$$
\begin{aligned}
& \mathrm{S} e_{2} \cdots \mathrm{Se}_{2}{ }_{2}{ }^{\prime \prime}=3.516(4) \\
& \mathrm{Se}_{2} \cdots \mathrm{Se}_{2}{ }^{\prime \prime \prime}=3.516(4) \\
& \mathrm{Se}_{1} \cdots \mathrm{Se}_{1}{ }^{\prime \prime}=3.567(8) \\
& \mathrm{Se}_{1} \cdots \mathrm{Se}_{1}{ }^{\prime \prime \prime}=3.567(8)
\end{aligned}
$$

$$
\begin{aligned}
& \angle \mathrm{C}_{1}-\mathrm{S}_{1} \cdots \mathrm{Se}_{1}{ }^{\prime \prime}=164.9(9) \\
& \angle \mathrm{C}_{1}-\mathrm{Se}_{1} \cdots \mathrm{Se}_{1}{ }^{\prime \prime \prime}=108.8(8) \\
& \angle \mathrm{C}_{2}-\mathrm{Se}_{2} \cdots \mathrm{Se}_{2}{ }^{\prime \prime}=98.6(14) \\
& \angle \mathrm{C}_{2}-\mathrm{Se}_{2} \cdots \mathrm{Se}_{2}{ }^{\prime \prime \prime}=174.6(14)
\end{aligned}
$$

Table 6. Distances from least squares planes. The equations of the planes were calculated with the selenium coordinates given six times the weight of the carbon and nitrogen coordinates, and refer to the axes of the unit cell, with coordinates $Y$ and $Z$ in $\AA$.

Plane through $\mathrm{Se}_{1} \mathrm{C}_{1} \mathrm{~N}_{1}, \mathrm{Se}_{2}$, and $\mathrm{Se}_{1}{ }^{\prime} \mathrm{C}_{1}{ }^{\prime} \mathrm{N}_{1}{ }^{\prime}$ :

$$
\begin{aligned}
& 0.8863 Y-0.4632 Z+2.0023=0 \\
& \begin{array}{lrlllll}
\mathrm{Se}_{1} & 0.024 \AA & \mathrm{C}_{1} & -0.003 \AA & \mathrm{N}_{1} & 0.000 \AA
\end{array}
\end{aligned}
$$

Plane through $\mathrm{Se}_{1}, \mathrm{Se}_{2} \mathrm{C}_{2} \mathrm{~N}_{2}$, and $\mathrm{Se}_{1}{ }^{\prime}$ :

$$
\begin{array}{cccccc}
\multicolumn{7}{c}{0.7943} & Y+0.6075 Z-2.7068=0 \\
\mathrm{Se}_{1} & -0.018 \AA & \mathrm{C}_{2} & 0.000 \AA & \mathrm{N}_{2} & 0.002 \AA \\
\mathrm{Se}_{2} & 0.009 & & & &
\end{array}
$$

Plane through $\mathrm{C}_{2}, \mathrm{Se}_{1} \mathrm{Se}_{2} \mathrm{Se}_{1}{ }^{\prime}$, and $\mathrm{Se}_{2}{ }^{\prime \prime \prime}$ :

$$
0.7293 Y+0.6842 Z-3.0274=0
$$

$$
\begin{array}{llllll} 
& & \mathrm{Se}_{1} & 0.021 \AA & & \\
\mathrm{C}_{2}-0.165 \AA & \mathrm{Se}_{2} & 0.000 & \mathrm{Se}_{2}{ }^{\prime \prime \prime} & 0.015 \AA
\end{array}
$$

Acta Chem. Scand. 25 (1971) No. 8 


\section{INTERIONIC SELENIUM - SELENIUM CONTACTS}

Each selenium atom in the three-selenium sequence has two close selenium selenium contacts to atoms in adjacent triselenocyanate ions. The relevant data are listed in Table 5. In the table, a double-prime denotes an atom located at $\left(x, \frac{1}{2}+y, \frac{1}{2}-z\right)$ relative to the unprimed one, and a triple-prime denotes an atom located at $\left(x, y-\frac{1}{2}, \frac{1}{2}-z\right)$ relative to the unprimed one; the double-primed and triple-primed atoms are both related to the unprimed ones through the operation of the mirror plane parallel to (100), passing through the middle selenium atom, $\mathrm{Se}_{2}$, followed by the operation of the screw axis parallel to $b$ near $\mathrm{Se}_{2}$.

Table 5 shows close $\mathrm{Se}_{2} \cdots \mathrm{Se}_{2}{ }^{\prime \prime}$ and $\mathrm{Se}_{2} \cdots \mathrm{Se}_{2}{ }^{\prime \prime \prime}$ contacts of $3.516(4) \AA$, and $\mathrm{Se}_{1} \cdots \mathrm{Se}_{1}{ }^{\prime \prime}$ and $\mathrm{Se}_{1} \cdots \mathrm{Se}_{1}{ }^{\prime \prime \prime}$ contacts of 3.567(8) $\AA$. For comparison, the Pauling ${ }^{9}$ van der Waals radius of selenium is $2.00 \AA$.

The $\mathrm{Se}_{2}{ }^{\prime \prime}$ atom approaches the fourth coordination site of square-planar four-coordination at $\mathrm{Se}_{2}$. Thus, the $\mathrm{Se}_{1} \mathrm{Se}_{2} \mathrm{Se}_{1}{ }^{\prime}, \mathrm{Se}_{2}{ }^{\prime \prime \prime}$, and $\mathrm{C}_{2}$ atoms lie approximately in the same plane ( $c f$. Table 6), the $\mathrm{Se}_{1}-\mathrm{Se}_{2}-\mathrm{Se}_{1}{ }^{\prime}$ angle is $176.9^{\circ}$, the $\mathrm{Se}_{2}-\mathrm{C}_{2}$ bond bisects the $\mathrm{Se}_{1}-\mathrm{Se}_{2}-\mathrm{Se}_{1}{ }^{\prime}$ angle, and the $\mathrm{C}_{2}-\mathrm{Se}_{2} \cdots \mathrm{Se}_{2}{ }^{\prime \prime \prime}$ angle is $174.6^{\circ}$. The same structural feature occurs in the crystals of the potassium salt. ${ }^{2}$

The $\mathrm{Se}_{2} \cdots \mathrm{Se}_{2}{ }^{\prime \prime}$ contact, also $3.516 \AA$, occurs at a $\mathrm{C}_{2}-\mathrm{Se}_{2} \cdots \mathrm{Se}_{2}{ }^{\prime \prime}$ angle of $98.6^{\circ}$.

The two close contacts of the terminal selenium atoms occur at C.-Se $\cdots \mathrm{Se}$ angles of $164.9^{\circ}$ and $108.8^{\circ}$.

\section{THE ENVIRONMENT OF THE RUBIDIUM ION AND THE WATER MOLECULE}

The water molecule lies in position of $m m$ symmetry at $(0, y, 0)$. It is surrounded by two rubidium ions at distances of $3.03(3) \AA$ and a $\mathrm{Rb} \cdots \mathrm{O} \cdots \mathrm{Rb}$ angle of $104.3(10)^{\circ}$, and by two $\mathrm{N}_{2}$ nitrogen atoms at distances of 3.09(5) $\AA$ and a $\mathrm{N} \cdots \mathrm{O} \cdots \mathrm{N}$ angle of $83.9(11)^{\circ}$. The latter contacts probably involve weak $\mathrm{O}-\mathrm{H} \cdots \mathrm{H}$ hydrogen bonds. The arrangement around the water molecule is approximately tetrahedral, the $\mathrm{Rb} \cdots \mathrm{O} \cdots \mathrm{N}$ angle being $117.2(6)^{\circ}$.

Table \%. Distances from the rubidium ion to neighbouring atoms. Standard deviation, $0.03 \AA$.

$\mathrm{Rb} \cdots \mathrm{N}_{1}(x, y, z)=3.10 \AA$
$\operatorname{Rb} \cdots \mathrm{N}_{1}(x, 1+y, z)=3.00$
$\operatorname{Rb} \cdots \mathrm{N}_{1}(x, y, \bar{z})=3.10$
$\operatorname{Rb} \cdots \mathrm{N}_{1}(x, 1+y, \bar{z})=3.00$

$\mathrm{Rb} \cdots \mathrm{N}_{\Omega}(x, y, z)=3.23 \AA$

$\mathrm{Rb} \cdots \mathrm{N}_{2}(x, y, \bar{z})=3.23$.

$\mathrm{Rb} \cdots \mathrm{O}^{2}=3.03$

The closest contacts of the rubidium ion are listed in Table 7 and shown in Fig. 2. The rubidium ion is surrounded by six nitrogen atoms at distances ranging from 3.00 to $3.23 \AA$, and by one oxygen atom, at $3.03 \AA$. Four of the six nitrogen atoms, at $\mathrm{Rb} \cdots \mathrm{N}=3.00$ and $3.10 \AA$, lie exactly in a plane, parallel to (100), at $x=0.1768$. The two remaining nitrogen atoms, at $\mathrm{Rb} \cdots \mathrm{N}=3.23 \AA$,

Acta Chem. Scand. 25 (1971) No. 8 


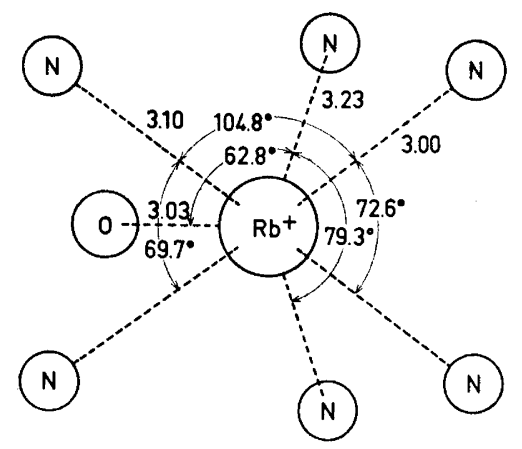

Fig. 2. The surroundings of the rubidium ion in rubidium triselenocyanate hemihydrate, as seen along the $a$ crystal axis.

and the oxygen atom, lie in the plane (100) at $x=0$. The rubidium ion lies $0.54 \AA$ from the former plane and $2.40 \AA$ from the latter plane, between the planes. The $R b \cdots O$ distance is in the normal range ( $c f$. Ref. 6, p. 259), and so are the $R b \cdots N$ distances, the sum of the $R b \cdots N$ ionic radii being $3.19 \AA .9$

\section{REFERENCES}

1. Verneuil, A. Ann. Chim. Phys. [6] 9 (1886) 289.

2. Hauge, S. and Sletten, J. Acta Chem. Scand. 25 (1971) 3094.

3. Birckenback, L. and Kellermann, K. Ber. 58 (1925) 2377.

4. Hauge, S. Acta Chem. Scand. 25 (1971) 3081.

5. Coppens, P., Leiserowitz, L. and Rabinovich, D. Acta Cryst. 18 (1965) 1035.

6. Zachariasen, W. H. Acta Cryst. 16 (1963) 1139.

7. International Tables for X-Ray Crystallography, Kynoch Press, Birmingham 1962, Vol. III.

8. Hauge, S., Opedal, O. and Aarskog, J. Acta Chem. Scand. 24 (1970) 1107.

9. Pauling, L. The Nature of the Chemical Bond, 3rd Ed., Cornell University Press, Ithaca, New York 1960.

10. Swank, D. D. and Willett, R. D. Inorg. Chem. 4 (1965) 499.

Received December 21, 1970. 